\title{
Does tourniquet use decrease blood loss following primary total knee arthroplasty in Jehovah's Witness patients?
}

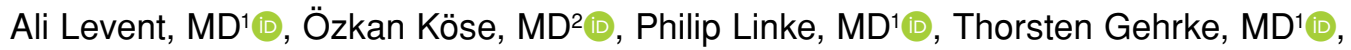 \\ Mustafa Çıtak, MD, $\mathrm{PhD}^{1}$ (D) \\ 'Department of Orthopedic Surgery, ENDO-Klinik Hamburg, Hamburg, Germany \\ ${ }^{2}$ Department of Orthopedics and Traumatology, University of Health Sciences, Antalya Training and Research Hospital, Antalya, Turkey
}

Total knee arthroplasty (TKA) is an effective solution for patients with end-stage knee osteoarthritis. ${ }^{[1]}$ Total knee arthroplasty may lead to serious blood loss that may necessitate a blood transfusion. During a TKA operation, up to 2,200 $\mathrm{mL}$ blood loss has been reported in previous studies. ${ }^{[2-5]}$ Various pre-, intra-, and postoperative strategies are used to reduce blood loss during TKA. ${ }^{[3]}$ Preoperative methods include iron therapy, administration of erythropoietin, and autologous blood donation. ${ }^{[3]}$ Intraoperative methods can be listed as hypotensive anesthesia, local or systemic tranexamic acid (TXA) administration, cell salvage, and tourniquet application. ${ }^{[6,7]}$ Blood loss and consequent anemia may have several undesirable effects both on mortality and morbidity, such as prolonged length of hospital stay, difficulty in achieving rehabilitation goals, and even death. Besides, blood transfusion carries many risks, including fatal

Received: May 16, 2020

Accepted: June 05, 2020

Published online: July 16, 2020

Correspondence: Ali Levent, MD. Department of Orthopedic Surgery, ENDO-Klinik Hamburg, Holstenstr. 2, 22767 Hamburg, Germany.

E-mail: dralilevent@hotmail.com

Doi: $10.5606 /$ ehc. 2020.76244

Citation: Levent A, Köse Ö, Linke P, Gehrke T, Çıtak M. Does tourniquet use decrease blood loss following primary total knee arthroplasty in Jehovah's Witness patients?. Jt Dis Relat Surg 2020;31(3):419-425

(O2020 All right reserved by the Turkish Joint Diseases Foundation

This is an open access article under the terms of the Creative Commons Attribution-NonCommercial License, which permits use, distribution and reproduction in any medium, provided the original work is properly cited and is not used for commercial purposes (http://creativecommons.org/licenses/by-nc/4.0/)

\section{ABSTRACT}

Objectives: This study aims to investigate the use of multiple blood management strategies and the effect of tourniquet on the estimated blood loss (EBL) in Jehovah's Witness (JW) patients who underwent primary total knee arthroplasty (TKA).

Patients and methods: Twenty-two self-reported JW patients (9 males, 13 females; mean age $66.8 \pm 8.6$ years; range, 51 to 83 years) who underwent primary TKA between January 2014 and January 2020 in our institution were retrospectively reviewed. A standard blood management protocol that consisted of hypotensive anesthesia, local and systemic administration of tranexamic acid (TXA) and intraoperative cell salvage was applied to all patients. Patients were divided into two groups: with $(n=11)$ and without $(\mathrm{n}=11)$ tourniquet use. The EBL was calculated according to Meunier's formula. Hemoglobin (Hgb), hematocrit (Hct), and EBL on the first and third postoperative days were compared statistically.

Results: There was no significant difference between groups regarding postoperative $\mathrm{Hgb}(\mathrm{p}=0.801$ and $\mathrm{p}=0.767)$, Hct $(\mathrm{p}=0.617$ and $\mathrm{p}=0.895)$, Hgb decline $(\mathrm{p}=0.311$ and $\mathrm{p}=0.822)$, and EBL $(p=0.067$ and $p=0.284)$ at first and third postoperative days. None of the patients required blood transfusion. No wound complication or symptomatic deep vein thrombosis was seen during the hospital stay.

Conclusion: Combined use of hypotensive anesthesia, intravenous administration of TXA, intraoperative periarticular injection, and cell salvage seem to be sufficient in controlling the blood loss in JW patients during TKA. Additional tourniquet use may not further decrease the EBL.

Keywords: Blood loss, blood transfusion, Jehovah's Witness, total knee arthroplasty, tourniquet.

anaphylactic reactions and transmission of infectious diseases. ${ }^{[8]}$

Due to their religious beliefs, Jehovah's Witnesses (JWs) do not allow blood transfusion, thus the use of blood management strategies, either alone or in combination, becomes more critical in these patients. ${ }^{[8-10]}$ However, there is no consensus or 
accepted algorithms about the methods to be used in the management of these patients. ${ }^{[1]}$ There are contradictory studies on whether tourniquet use reduces blood loss in TKA. ${ }^{[12-14]}$ In essence, to our knowledge, there are no studies examining the effect of tourniquet use on blood loss during TKA in JWs in the current literature. ${ }^{[15]}$ Therefore, to overcome these shortcomings, we aimed to answer the following questions: (i) Does the use of tourniquet decrease the amount of blood loss during primary TKA? (ii) Is the use of tourniquet associated with higher complication rates following primary TKA? Thus, in this study, we aimed to investigate the use of multiple blood management strategies and the effect of tourniquet on the estimated blood loss (EBL) in JW patients who underwent primary TKA.

\section{PATIENTS AND METHODS}

A retrospective review was performed on the digital medical database to identify all self-reported JW patients who underwent primary TKA between January 2014 and January 2020 in Department of Orthopedic Surgery, ENDO-Klinik, Hamburg. Patients with incomplete or inadequate medical records were excluded from the study. Patients with a history of hematological or coagulation disorders and thromboembolic events were also excluded. A total of 25 eligible patients were identified during the period under investigation, while three patients were excluded due to missing clinical records. Finally, 22 patients ( 9 males, 13 females; mean age $66.8 \pm 8.6$ years; range, 51 to 83 years) who had complete medical records were included. The study protocol was approved by the General Medical Council Hamburg Ethics Committee (6.5.2020/WF-069/20). A written informed consent was obtained from each patient. The study was conducted in accordance with the principles of the Declaration of Helsinki.

None of the patients received preoperative blood loss prevention methods such as iron therapy, erythropoietin (EPO) administration, or autologous blood donation, because there were no patients who had preoperative hemoglobin $(\mathrm{Hgb})$ less than $10 \mathrm{gr} / \mathrm{dL}$. All patients underwent the TKA procedure under general anesthesia. The following blood management strategies were used in combination with all patients. (i) All patients underwent hypotensive anesthesia, and mean arterial pressure was kept below $65 \mathrm{mmHg}$ during the operation. (ii) TXA $1 \mathrm{~g}$ intravenous (IV) was given 15 minutes prior to surgical incision. (iii) After completing the bone-cuts, $222 \mathrm{~mL}$ periarticular injection (Table I) in all the soft tissues surrounding the knee joint, including posterior capsule, collateral ligaments, peritendinous structures, and subcutaneous tissue was performed. (iv) A continuous autologous transfusion system (CATS, Fresenius AG, Bad Homburg, Germany) was used in all patients. This auto-transfusion system prepared the shed blood through the separation of corpuscular particles, washing of concentrated erythrocytes with heparinized saline solution and separation of erythrocytes, and consumed washing solution. The auto-transfused unit contains about $150 \mathrm{~mL}$ prepared red cells, and $30 \mathrm{~g}$ of $\mathrm{Hgb}$ was administered within six hours postoperatively. ${ }^{[16]}$

Four different blood management strategies mentioned above were applied to all patients. However, the tourniquet was applied to 11 patients (tourniquet group), and TKA was performed without a tourniquet in the remaining 11 patients (non-tourniquet group). TKA is almost routinely performed without tourniquets in authors' institution. Decision to use or not to use tourniquet was the surgeon's personal preference in this series. The tourniquet was inflated to a pressure of $320 \mathrm{mmHg}$ and released just after the placement of the components. Meticulous coagulation was carried out throughout the whole procedure. No suction drain was used in any of the patients. A posterior stabilized knee prosthesis (NexGen ${ }^{\circledR}$ LPS-Flex Fixed Bearing Knee, Zimmer-Biomet, Warsaw, Indiana, USA) was used in all patients.

Age, sex, weight, height, body mass index (BMI), Charlson comorbidity index, American Society of Anesthesiologists (ASA) score, preoperative complete blood count $(\mathrm{CBC})$ including $\mathrm{Hgb}$, hematocrit (Hct), and international normalized ratio (INR) were recorded. Postoperative $\mathrm{CBC}$ on the first and third days was recorded. Total blood volume was calculated according to Nadler's formula based on sex, height, and weight of the patient. ${ }^{[17]}$ The EBL was calculated according to Meunier's formula (Table II). ${ }^{[17,18]}$ Estimated blood loss and $\mathrm{Hgb}$ decline in the first and third postoperative days were analyzed and compared between both groups. Length of hospital stay and all in-hospital complications were recorded in all patients. $\mathrm{Hgb}<6 \mathrm{gr} / \mathrm{dL}$ or $\mathrm{Hgb}<8 \mathrm{gr} / \mathrm{dL}$ with hypotension, tachycardia, or signs of heart failure

\begin{tabular}{|lcc|}
\multicolumn{3}{c}{ TABLE I } \\
Composition of periarticular injection \\
\hline Medication & Dose/strength & Amount $(\mathrm{mL})$ \\
\hline Diclofenac sodium & $75 \mathrm{mg}$ & 2 \\
Tranexamic acid & $2 \mathrm{~g}$ & 20 \\
Ropivacaine hydrochloride & $400 \mathrm{mg}$ & 200 \\
Total & & 222 \\
\hline
\end{tabular}




\begin{tabular}{|c|c|}
\hline \multicolumn{2}{|c|}{$\begin{array}{l}\text { TABLE II } \\
\text { Formulas used for calculation of total blood volume and estimated blood loss }\end{array}$} \\
\hline BV calculation & Formula \\
\hline \multicolumn{2}{|l|}{ Sex } \\
\hline Male & $\left(0.3669 \times\right.$ Height $\left.^{3}\right)+(0.03219 \times$ Weight $)+0.6041$ \\
\hline Female & $\left(0.3561 \times\right.$ Height $\left.^{3}\right)+(0.03308 \times$ Weight $)+0.1833$ \\
\hline Estimated blood loss calculation & $B V \times\left[\left(\mathrm{Hgb}_{i}-\mathrm{Hgb}_{\mathrm{f}}\right) / \mathrm{Hgb}_{\mathrm{i}}\right]$ \\
\hline
\end{tabular}

was set as transfusion indication according to the institution's blood transfusion protocol after TKA.

As part of the thrombophylaxis protocol, all patients received daily 0.4 IU low-molecular-weight heparin started at eight hours postoperatively.

\section{Statistical analysis}

Continuous variables were presented as mean \pm standard deviation and range. Categorical variables were presented as frequency distribution. The Kolmogorov-Smirnov test was used to determine whether the data were distributed normally. The effect of using a tourniquet on EBL was analyzed in both groups. A comparative analysis of two independent groups was performed using the Mann-Whitney $U$ test and the Student's t-test. A value of $p<0.05$ was accepted as statistically significant.

\section{RESULTS}

Both study groups were similar regarding baseline demographic and clinical characteristics including age $(p=0.134)$, height $(p=0.171)$, weight $(p=0.481)$, BMI $(p=0.880)$, Charlson score $(p=0.300)$, ASA score $(p=0.665)$, length of hospital stay $(p=0.133)$, preoperative INR $(\mathrm{p}=0.127)$, Hgb $(\mathrm{p}=0.640)$, Hct $(\mathrm{p}=0.548)$, and total blood volume $(\mathrm{p}=0.150)$ except for sex distribution $(p=0.004)$ (Table III). On the first postoperative day, EBL and Hgb decline were slightly higher in the tourniquet group, but the difference was not statistically significant $(p=0.067$ and $\mathrm{p}=0.311$, respectively). There was no significant difference between groups regarding postoperative $\mathrm{Hgb}$, Hct, Hgb loss, and EBL (Table IV). Hemoglobin was found to be $7.5 \mathrm{gr} / \mathrm{dL}$ in only one patient on the third postoperative day. However, no clinical findings

\begin{tabular}{|c|c|c|c|c|c|}
\hline \multicolumn{6}{|c|}{$\begin{array}{l}\text { TABLE III } \\
\text { ic and clinical characteristics of patients }\end{array}$} \\
\hline & \multicolumn{2}{|c|}{$\begin{array}{l}\text { Group } 1 \\
\text { Tourniquet }(n=11)\end{array}$} & \multicolumn{2}{|c|}{$\begin{array}{l}\text { Group } 2 \\
\text { No tourniquet }(n=11)\end{array}$} & \multirow{2}{*}{$\begin{array}{c}\text { Significance } \\
p\end{array}$} \\
\hline & $\mathrm{n}$ & Mean $\pm S D$ & $n$ & Mean $\pm S D$ & \\
\hline Age (year) & & $69.6 \pm 10.4$ & & $64.0 \pm 5.3$ & 0.134 \\
\hline Sex & & & & & $0.004^{*}$ \\
\hline Male & 8 & & 1 & & \\
\hline Female & 3 & & 10 & & \\
\hline Height $(\mathrm{cm})$ & & $173.0 \pm 9.1$ & & $167.1 \pm 9.4$ & $0.171 \dagger$ \\
\hline Weight (kg) & & $98.1 \pm 12.8$ & & $93.0 \pm 19.6$ & 0.481 \\
\hline Body mass index $\left(\mathrm{kg} / \mathrm{m}^{2}\right)$ & & $32.7 \pm 5.3$ & & $33.1 \pm 6.0$ & 0.880 \\
\hline Charlson score (point) & & $4.1 \pm 1.2$ & & $3.4 \pm 1.5$ & $0.300 \dagger$ \\
\hline \multicolumn{6}{|l|}{ ASA } \\
\hline II & 6 & & 5 & & \\
\hline III & 5 & & 6 & & \\
\hline Preoperative INR (value) & & $1.0 \pm 0.0$ & & $1.0 \pm 0.1$ & 0.127 \\
\hline Preoperative $\mathrm{Hgb}$ (gr/dL) & & $13.9 \pm 1.4$ & & $13.6 \pm 1.2$ & 0.640 \\
\hline Preoperative Hct (\%) & & $39.3 \pm 4.3$ & & $40.3 \pm 3.2$ & 0.548 \\
\hline Blood volume (mL) & & $3674.5 \pm 474.6$ & & $3293.0 \pm 699.3$ & 0.150 \\
\hline
\end{tabular}




\begin{tabular}{|c|c|c|c|}
\hline \multicolumn{4}{|c|}{$\begin{array}{c}\text { TABLE IV } \\
\text { Comparison of hematological characteristics between groups }\end{array}$} \\
\hline & Tourniquet $(n=11)$ & No tourniquet $(n=11)$ & Significance \\
\hline Variable & Mean \pm SD & Mean \pm SD & $p$ \\
\hline Postoperative INR at $1^{\text {st }}$ day (value) & $1.0 \pm 0.04$ & $1.0 \pm 0.08$ & 0.400 \\
\hline Postoperative Hgb at $1^{\text {st }}$ day $(\mathrm{gr} / \mathrm{dL})$ & $11.0 \pm 1.1$ & $11.2 \pm 1.6$ & 0.801 \\
\hline Postoperative $\mathrm{Hgb}$ at $3^{\text {rd }}$ day $(\mathrm{gr} / \mathrm{dL})$ & $10.6 \pm 1.7$ & $10.4 \pm 1.2$ & 0.767 \\
\hline Hgb decline at $1^{\text {st }}$ day ( $\left.g r / d L\right)$ & $2.8 \pm 0.86$ & $2.4 \pm 1.05$ & 0.311 \\
\hline Hgb decline at $3^{\text {rd }}$ day $(\mathrm{gr} / \mathrm{dL})$ & $3.2 \pm 0.8$ & $3.1 \pm 0.8$ & 0.822 \\
\hline Post-operative Hct at $1^{\text {st }}$ day (\%) & $32.1 \pm 3.4$ & $33.0 \pm 4.9$ & 0.617 \\
\hline Post-operative Hct at $3^{\text {rd }}$ day (\%) & $30.8 \pm 4.6$ & $31.1 \pm 3.5$ & 0.895 \\
\hline Estimated blood loss at $1^{\text {st }}$ day $(\mathrm{mL})$ & $749.6 \pm 221.2$ & $571.3 \pm 209.6$ & 0.067 \\
\hline Estimated blood loss at $3^{\text {rd }}$ day $(\mathrm{mL})$ & $865.5 \pm 304.5$ & $750.9 \pm 162.3$ & 0.284 \\
\hline Hospital stay (days) & $7.7 \pm 1.4$ & $6.7 \pm 1.4$ & $0.133^{*}$ \\
\hline
\end{tabular}

were observed in this patient. Hemoglobin levels did not fall to the transfusion threshold in any other patient (minimum: $8.6 \mathrm{gr} / \mathrm{dL}$ ). None of the patients were followed-up in the intensive care unit, and no complications were seen in any of the patients, such as wound complications or symptomatic deep vein thrombosis during the hospital stay. The length of hospital stay was similar between groups $(p=0.133)$.

\section{DISCUSSION}

The most important finding of our study was that tourniquet use did not decrease blood loss following primary TKA in JW patients. However, it should be noted that the combined use of hypotensive anesthesia, local and systemic administration of TXA, and intraoperative cell salvage (ICS) provided safe and effective bleeding control. When all the strategies of this blood management protocol are followed, additional use of tourniquet does not have any statistically significant effect on the amount of blood loss. Orthopedic surgeons must be careful during major surgical procedures such as primary TKA in JW patients, and all blood loss prevention measures should be taken whenever possible to avoid life-threatening anemia. Our protocol described here may be a suitable option for surgeons who do not want to use a tourniquet.

Cell salvage may be performed either intra- or postoperatively or as a combination of both in TKA. While ICS collects the blood from the surgical site, postoperative cell salvage (PCS) collects the blood accumulated in the suction drain. In both methods, collected blood passes through several filters and preparation steps, and then the blood is returned via a standard setting within six hours postoperatively. In this series, no suction drain was used in any patient, and PCS was not performed. ICS was started at the beginning of the operation in patients without a tourniquet, and after the tourniquet release in patients with a tourniquet. Since the tourniquet was released immediately after the placement of components, a considerable amount of blood could be prepared until the wound closure in all patients. There are contradictory findings in the literature regarding the use of cell salvage in TKA. Schneider et al. ${ }^{[19]}$ reported that cell salvage had no beneficial effect on the rate of allogeneic blood transfusion. In contrast, Park et al. ${ }^{[20]}$ showed a significant reduction in blood transfusion in their study. Some authors have also claimed that this practice is not cost-effective. ${ }^{[21]}$ In a recent meta-analysis, 19 randomized clinical trials were evaluated, and it was concluded that the need for blood transfusion was significantly reduced during TKA using cell salvage. ${ }^{[22]}$ However, the different transfusion protocols for each of the included studies compromise the reliability of the withdrawals. Although there is no strong evidence-based data on its efficacy, we believe that cell salvage should be included in the bleeding control protocol in JW patients. Suh et al. ${ }^{[11]}$ have developed a bleeding control protocol that consists of pre- and postoperative EPO and iron therapy, use of tourniquet, hemodilution with plasma expander, and cell salvage for JW patients planned for TKA. Seventy-seven TKAs were performed in accordance with this protocol, and none of the patients required blood transfusion. ${ }^{[1]}$ Although there are some similarities between our protocol and 
the protocol in the above-mentioned study, there are also critical differences. The use of cell salvage in both protocols is the only common aspect; however, in this study, PCS was utilized because tourniquet and suction drain were used. Since none of our patients had preoperative hemoglobin below $10 \mathrm{mg} / \mathrm{dL}, \mathrm{EPO}$ or iron therapy was not administered in any patient. In half of our patients, a tourniquet was not used, and we found that this had no effect on EBL. The most important difference between our protocol from this protocol is the use of systemic and local TXA. Apart from this clinical study, Panousis et al. ${ }^{[23]}$ reported a 55-year-old JW patient who had rheumatoid arthritis and had undergone one-stage ipsilateral TKA and total hip arthroplasty. Furthermore, the patient had borderline anemia. Similarly, the patient was managed with preoperative iron therapy, cell salvage, and intraoperative controlled hypotension. After six weeks, the same procedure was performed for the contralateral side. Although the patient had four major joint replacements within six weeks, blood transfusion was not required, and no major complications were seen. Although the blood management protocol has been properly implemented, we think that simultaneous joint replacement is risky and should not be recommended for JW patients.

There are several studies supporting that TXA is useful for the prevention of blood loss in TKA. Thus, it became a routine practice in almost all TKA patients unless there is a contraindication. However, controversy still goes on about its total dose, timing, and route of application. ${ }^{[24-30]}$ However, in a recent meta-analysis, the combined use of TXA (both IV and topical) has been shown to be more effective over IV use only. ${ }^{[3]}$ Consistently, a combined TXA application was carried out in our protocol. However, topical TXA was prepared as a cocktail together with ropivacaine hydrochloride and diclofenac sodium and applied not only by pouring it into the wound but through periarticular injection. Ropivacaine is a long-acting local anesthetic agent that provides postoperative analgesia, and diclofenac sodium reduces local inflammation. There are also studies reporting additional benefits of TXA reducing inflammation and infection risk in TKA. ${ }^{[2,27]}$

In the current literature, there are few other descriptions of topical use of TXA mixed with other ingredients. In a study conducted by Tandogan et al., ${ }^{[29]}$ TXA was combined with platelet-rich fibrin. However, no significant reduction in blood loss was found compared to the sole use of TXA. Stamatopoulos et al. ${ }^{[32]}$ used IV TXA and a topical mixture of TXA with adrenalin. They reported significant bleeding control with their protocol. Because of our concerns about the consequences of vasoconstriction on wound healing, adrenalin was not added in our cocktail. There is another famous periarticular mixture described by the Ranawat Orthopaedics Centre, also known as ROC cocktail with multiple ingredients and effects. ROC cocktail consists of bupivacaine, morphine sulphate, epinephrine, methylprednisolone acetate, cefuroxime, and saline. ${ }^{[33]}$ In fact, the main purpose of the ROC cocktail is to provide postoperative pain control, but it also reduces bleeding through vasoconstriction provided by epinephrine. ${ }^{[33]}$ Similar to diclofenac sodium in our cocktail, the ROC cocktail includes steroids, which are potent antiinflammatory agents, providing local inflammation and preventing the side effects of morphine sulphate.

Tourniquet use is still one of the controversial issues in TKA. In a recent meta-analysis that included 18 studies $(n=1,279)$, tourniquet use was associated with increased postoperative pain, decreased postoperative range of motion, and increased rate of deep venous thrombosis. ${ }^{[34]}$ In another metaanalysis that evaluated seven studies $(n=505)$, there was no significant difference in the need for blood transfusion between the group using the tourniquet and the control group. ${ }^{[35]}$ Tetro and Rudan ${ }^{[36]}$ have even reported a larger amount of blood loss $(p>0.05)$ in the tourniquet group (1792 cc versus $1499 \mathrm{cc}$, respectively). Similar to this study, in our study, the blood loss in the tourniquet group was slightly higher than the non-tourniquet group, but without a statistically significant difference. In light of the findings of our study, if all other steps of the protocol are used together in JW patients, TKA may be performed without using a tourniquet.

There are some limitations to this study. First, it was a retrospective case series conducted on a limited number of patients without random assignment. Second, the data included only the early postoperative period. However, the main goal of our study was to analyze the blood management strategies. Third, the study lacked a control group. To mention the strengths of this study, both groups had similar preoperative features suitable for comparison. Except for the use of a tourniquet, the same protocol was applied to all patients, and all were operated by the same surgical team.

In conclusion, the protocol described in this study has enabled us to perform TKA without postoperative blood transfusion and postoperative complications in JW patients. Furthermore, TKA may be performed without using a tourniquet in JW patients by using 
the described protocol. Surgeons should be cautious when performing TKA in a JW patient and take all necessary measures for bleeding control.

\section{Declaration of conflicting interests}

The authors declared no conflicts of interest with respect to the authorship and/or publication of this article.

\section{Funding}

The authors received no financial support for the research and/or authorship of this article.

\section{REFERENCES}

1. Themistoklis T, Theodosia V, Konstantinos K, Georgios DI. Perioperative blood management strategies for patients undergoing total knee replacement: Where do we stand now? World J Orthop 2017;8:441-54.

2. Knight JL, Sherer D, Guo J. Blood transfusion strategies for total knee arthroplasty: minimizing autologous blood wastage, risk of homologous blood transfusion, and transfusion cost. J Arthroplasty 1998;13:70-6.

3. Liu D, Dan M, Martinez Martos S, Beller E. Blood Management Strategies in Total Knee Arthroplasty. Knee Surg Relat Res 2016;28:179-87.

4. Noticewala MS, Nyce JD, Wang W, Geller JA, Macaulay W. Predicting need for allogeneic transfusion after total knee arthroplasty. J Arthroplasty 2012;27:961-7.

5. Mylod AG Jr, France MP, Muser DE, Parsons JR. Perioperative blood loss associated with total knee arthroplasty. A comparison of procedures performed with and without cementing. J Bone Joint Surg [Am] 1990;72:1010-2.

6. Lu Q, Peng H, Zhou GJ, Yin D. Perioperative blood management strategies for total knee arthroplasty. Orthop Surg 2018;10:8-16.

7. Cai DF, Fan QH, Zhong HH, Peng S, Song H. The effects of tourniquet use on blood loss in primary total knee arthroplasty for patients with osteoarthritis: a metaanalysis. J Orthop Surg Res 2019;14:348.

8. Harwin SF, Pivec R, Naziri Q, Issa K, Mont MA. Is total hip arthroplasty a successful and safe procedure in Jehovah's Witnesses? Mean five-year results. Hip Int 2014;24:69-76.

9. Harwin SF, Issa K, Naziri Q, Pivec R, Johnson AJ, Mont MA. Excellent results of revision TKA in Jehovah's Witness patients. J Knee Surg 2013;26:151-4.

10. Remmers PA, Speer AJ. Clinical strategies in the medical care of Jehovah's Witnesses. Am J Med 2006;119:1013-8.

11. Suh YS, Nho JH, Choi HS, Ha YC, Park JS, Koo KH. A protocol avoiding allogeneic transfusion in joint arthroplasties. Arch Orthop Trauma Surg 2016;136:1213-26.

12. Abdel-Salam A, Eyres KS. Effects of tourniquet during total knee arthroplasty. A prospective randomised study. J Bone Joint Surg [Br] 1995;77:250-3.

13. Zhang W, Li N, Chen S, Tan Y, Al-Aidaros M, Chen L. The effects of a tourniquet used in total knee arthroplasty: a meta-analysis. J Orthop Surg Res 2014;9:13.

14. Vandenbussche E, Duranthon LD, Couturier M, Pidhorz L, Augereau B. The effect of tourniquet use in total knee arthroplasty. Int Orthop 2002;26:306-9.

15. Issa K, Banerjee S, Rifai A, Kapadia BH, Harwin SF, McInerney VK, et al. Blood management strategies in primary and revision total knee arthroplasty for Jehovah's Witness patients. J Knee Surg 2013;26:401-4.
16. Hawi N, Kendoff DO, Hessling U, Haasper C, Gehrke T, Citak M. Effectiveness of an autologous transfusion system following cemented and non-cemented revisions of total hip arthroplasty. Int Orthop 2014;38:1603-8.

17. Nadler SB, Hidalgo JH, Bloch T. Prediction of blood volume in normal human adults. Surgery 1962;51:224-32.

18. Meunier A, Petersson A, Good L, Berlin G. Validation of a haemoglobin dilution method for estimation of blood loss. Vox Sang 2008;95:120-4.

19. Schneider MM, Kendoff D, Oloughlin PF, Hessling C, Gehrke T, Citak M. Effectiveness of autologous transfusion system in primary total hip and knee arthroplasty. Technol Health Care 2014;22:123-8.

20. Park KH, Lee SR, Jin JM, Moon MS. The efficacy and safety of postoperative autologous transfusion of filtered shed blood and anticoagulant prophylaxis in total knee arthroplasty patients. Knee Surg Relat Res 2012;24:14-8.

21. Rizzi L, Bertacchi P, Ghezzi LM, Bellavita P, Scudeller G. Postoperative blood salvage in hip and knee arthroplasty. A prospective study on cost effectiveness in 161 patients. Acta Orthop Scand 1998;69:31-4.

22. Xie J, Feng X, Ma J, Kang $P$, Shen B, Yang J, et al. Is postoperative cell salvage necessary in total hip or knee replacement? A meta-analysis of randomized controlled trials. Int J Surg 2015;21:135-44.

23. Panousis K, Rana B, Hunter J, Grigoris P. Rapid sequence quadruple joint replacement in a rheumatoid Jehovah'ss Witness. Arch Orthop Trauma Surg 2003;123:128-31.

24. Sezgin EA, Atik OŞ. Are we scientifically ready to adopt tranexamic acid as a routine in arthroplasty? Eklem Hastalik Cerrahisi 2019;30:338-9.

25. Nemoto A, Mizuno K, Goyagi T. The Effect of Tranexamic Acid Administration on Perioperative Bleeding in Patients Undergoing Knee or Hip Arthroplasty: A SingleCentre Retrospective Study. Turk J Anaesthesiol Reanim 2020;48:142-7.

26. Lei Y, Xie J, Huang Q, Huang W, Pei F. Additional benefits of multiple-dose tranexamic acid to anti-fibrinolysis and anti-inflammation in total knee arthroplasty: a randomized controlled trial. Arch Orthop Trauma Surg 2020. [Online ahead of print]

27. Lacko M, Jarčuška P, Schreierova D, Lacková A, Gharaibeh A. Tranexamic acid decreases the risk of revision for acute and delayed periprosthetic joint infection after total knee replacement. Jt Dis Relat Surg 2020;31:8-13.

28. Morales Santias M, Mas Martinez J, Sanz-Reig J, Martínez Gimenez E, Verdu Román C, Bustamante Suarez de Puga D. Topical tranexamic acid in cemented primary total knee arthroplasty without tourniquet: a prospective randomized study. Eur J Orthop Surg Traumatol 2020. [Online ahead of print]

29. Tandogan RN, Polat M, Beyzadeoglu T, Karabulut E, Yildirim K, Kayaalp A. Topical co-delivery of platelet rich fibrin and tranexamic acid does not decrease blood loss in primary total knee arthroplasty compared to the standard of care: a prospective, randomized, controlled trial. Knee Surg Sports Traumatol Arthrosc 2020. [Online ahead of print]

30. Tzatzairis T, Drosos GI, Vogiatzaki T, Tilkeridis K, Ververidis A, Kazakos K. Multiple intravenous tranexamic acid doses in total knee arthroplasty without tourniquet: a randomized controlled study. Arch Orthop Trauma Surg 2019;139:859-68. 
31. Wang Z, Shen X. The efficacy of combined intra-articular and intravenous tranexamic acid for blood loss in primary total knee arthroplasty: A meta-analysis. Medicine (Baltimore) 2017;96:e8123.

32. Stamatopoulos A, Stamatopoulos T, Kenanidis E, Potoupnis M, Sayegh F, Tsiridis E. Intravenous and intraarticular tranexamic acid plus epinephrine for the man-agement of blood loss after cemented total knee arthroplasty: a case-control study. Hippokratia 2018;22:86-90.

33. Maheshwari AV, Blum YC, Shekhar L, Ranawat AS, Ranawat CS. Multimodal pain management after total hip and knee arthroplasty at the Ranawat Orthopaedic Center. Clin Orthop Relat Res 2009;467:1418-23.

34. Jawhar A, Skeirek D, Stetzelberger V, Obertacke U. Influence of the Tourniquet on Pain and Function in Total Knee Arthroplasty: a Systematic Review and Meta-Analysis. Z Orthop Unfall 2019. [Online ahead of print]

35. Yi S, Tan J, Chen C, Chen H, Huang W. The use of pneumatic tourniquet in total knee arthroplasty: a meta-analysis. Arch Orthop Trauma Surg 2014;134:1469-76.

36. Tetro AM, Rudan JF. The effects of a pneumatic tourniquet on blood loss in total knee arthroplasty. Can J Surg 2001;44:33-8. 